\title{
The nature of the Galactic bulge: A view from bulge planetary nebulae and globular clusters
}

\section{Alexander F. Kholtygin, Yulia V. Milanova, Igor' I. Nikiforov and Olga V. Vasyakina}

\author{
Sobolev Astronomical Institute, Saint Petersburg State University, \\ Universitetskij pr. 28, Staryj Peterhof, Saint Petersburg 198504, Russia \\ email: afkholtygin@gmail.com
}

\begin{abstract}
Modern data concerning the planetary nebulae (PNe) in the bulge, bar and disk of the Milky Way are used to study the chemical history of bulge. We show that the abundance pattern is similar for PNe in the bulge and Peimbert's type II PNe. We also found that the globular clusters (GCs), especially their metal-rich disk subsystem, form on metallicity maps a bar-like structure which parameters are very close to those for the Galactic bar. These results evidence an old age of the Galactic bulge and bar. We propose a scenario of the successive star formation in the bulge, bar and thin disk.
\end{abstract}

Keywords. Planetary nebulae: general, globular clusters: general, Galaxy: bulge

\section{Nature of the bulge from PNe}

We choose the following criteria for selection of the bulge PNe: $l \leqslant 10^{\circ}, b \leqslant 8^{\circ}$, $F(5 \mathrm{GHz}) \leqslant 100 \mathrm{mJy}, R \leqslant 2 \mathrm{kpc}$. Here $l$ and $b$ are the Galactic longitude and latitude, $F(5 \mathrm{GHz})$ is the total radio flux from the $\mathrm{PN}$ at $5 \mathrm{GHz}$, and $R$ is the distance of the PN from the Galactic rotation axis. The values of $R$ were calculated from distances to $\mathrm{PNe}$ given by Akimkin et al. (2011). The last condition $(R \leqslant 2 \mathrm{kpc})$ is adopted to avoid the inclusion of objects close to the Sun to the list of the bulge PNe.

The nature of Galactic bulge remains not clearly understood up to now. The analysis of elemental abundances in the Galactic subsystems can illuminate this question. For all PNe of our sample the abundances were determined by the same way as in Milanova \& Kholtygin (2009). For the PNe of all types by Quireza et al. (2007), we obtained the mean abundances of $\mathrm{He}, \mathrm{C}, \mathrm{N}, \mathrm{O}, \mathrm{Ne}, \mathrm{S}, \mathrm{Ar}, \mathrm{Cl}$, and Fe. We compared these abundances with ones for objects of other Galactic subsystems. Our analysis shows that the abundances of bulge PNe are most similar to ones of type II (subtype IIa) PNe. We calculated the rms deviations $\sigma_{\mathrm{V}}=\left[\sum_{X}\left([X / \mathrm{H}]_{\mathrm{V}}-[X / \mathrm{H}]_{\text {type }}\right)^{2}\right]^{1 / 2}$ of mean relative abundances $[X / \mathrm{H}]$ for PNe in the bulge (type $\mathrm{V}$ ) from values of $[X / \mathrm{H}]$ for another Galactic type of PNe, where $X=\mathrm{He}, \mathrm{C}, \mathrm{N}, \mathrm{O}, \mathrm{Ne}, \mathrm{S}, \mathrm{Cl}, \mathrm{Ar}$, and Fe, as a function of the PNe type. We found that the minimal value of $\sigma_{\mathrm{V}}$ is achieved for PNe of type II, more exactly for subtype IIa.

We also established that the oxygen abundances in the bulge PNe continue the dependence of $[\mathrm{O} / \mathrm{H}]$ vs. Galactocentric distance $R$ for thin disk PNe.

Finally, we propose that the bulge is formed on an early stage of the Galaxy evolution in accordance with the Immeli et al. (2004) scenario. After that the second infall occurred at 4-6 Gyr before the modern era. This infall forms the thin disk finally. The intense enrichment of ISM in the bulge probably continues up to the present days. 

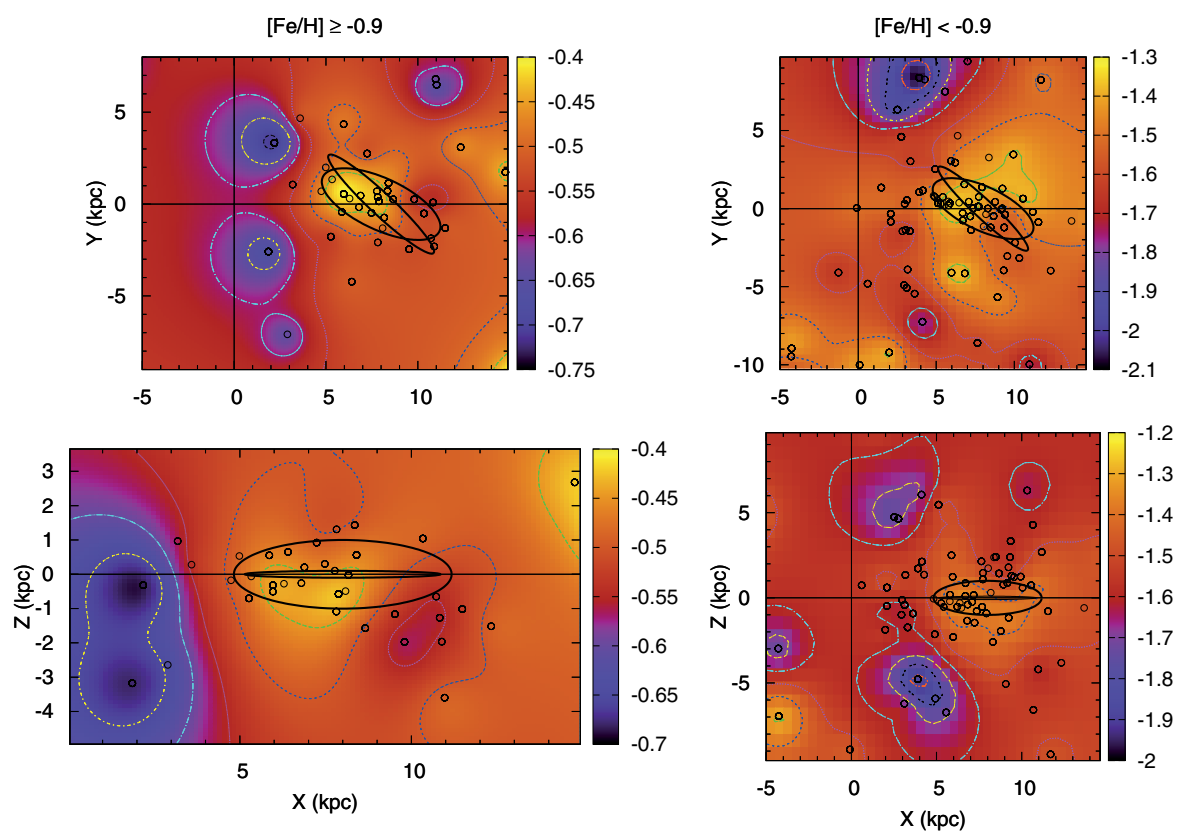

Figure 1. Metallicity maps for metal-rich (left) and metal-poor (right) subsystems of GCs. Open circles represent individual GCs. The thick solid lines confine the Galactic bar $(a=3.5 \mathrm{kpc}$, $b=1.4 \mathrm{kpc}$, an angle of $\left.25^{\circ}\right)$ and the long bar $(a=3.9 \mathrm{kpc}, b=0.6 \mathrm{kpc}$, an angle of $\left.43^{\circ}\right)$; see Gardner \& Flynn $(2010)$. The Sun is at $(X, Y)=(0,0)$, the Galactic center is at $(X, Y)=(8.0,0)$.

\section{Manifestation of the bar on metallicity maps of the GC subsystems}

We used data on GCs from the 2010 December version (hereafter H10) of the Harris (1996) catalogue, which provides values of $[\mathrm{Fe} / \mathrm{H}]$ for $152 \mathrm{GCs}$. We found that the division between the disk and halo $\mathrm{GC}$ subsystems is at $[\mathrm{Fe} / \mathrm{H}]=-0.9$ for the new $[\mathrm{Fe} / \mathrm{H}]$ scale.

For these GC subsystems, we have obtained the metallicity maps which, as well as the space distribution on the $X Y$ plane, evidence a centrally concentrated, bar-like configuration with parameters closely agreed with those for the Galactic bar (Fig. 1, top panels). This "bar subsystem" of GCs, being more pronounced for metal-rich GCs, shows itself also for metal-poor GCs. For both GC subsystems, the more metal-rich GCs fall always or mostly within the near side of the bar, in the far side GCs are not visible probably because of high extinction (Fig. 1, bottom panels).

This effect can suggest that the bar GCs were formed within the already existing Galactic bar. Hence it follows that the age of the Galactic bar may be 10 Gyr or more. So our findings evidence the generation of both the bulge and the bar on early stages of the Galaxy evolution.

\section{References}

Akimkin, V. V., Nikiforov, I. I., \& Kholtygin, A. F. MNRAS, in preparation

Gardner, E. \& Flynn, C. 2010, MNRAS, 405, 545

Harris, W. E. 1996, AJ, 112, 1487; arXiv:1012.3224 (2010 edition)

Immeli, A., Samland, M., \& Gerhard, O. 2004, A\& A, 413, 547

Milanova, Yu. V. \& Kholtygin, A. F. 2009, Astron. Lett., 35, 518

Quireza, C., Rocha-Pinto, H. J., \& Maciel, W. J. 2007, A\&SA, 475, 217 